Recepción: 01 / 03/ 2018

Aceptación: 15 / 05 / 2018

Publicación: 07 / 07 / 2018

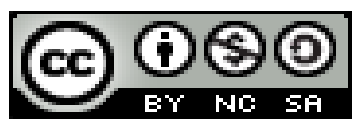

Ciencias de la Salud

Artículo de Investigación

\title{
Condiciones Ambientales y Leishmaniasis en el Cantón Jipijapa
}

Environmental Conditions and Leishmaniasis in the Jipijapa Canton

\section{Condições ambientais e leishmaniose no cantão de Jipijapa}

\author{
Rosa del Rocío Pinargote-Chancay ${ }^{\mathrm{I}}$ \\ Carlos A. Riofrio-Pinargote ${ }^{\text {IV }}$ \\ angel.m.86@hotmail.com \\ marina_b@hotmail.es \\ Noralma K. Jaime-Hernández II \\ Jennifer K. Cañarte-Mero v \\ narda.merchan@unesumedu.ec \\ erick.salazar@unesum.edu.ec \\ Margoth E. Villegas-Chiriboga III \\ Carmen A. Condo-Baque VI \\ amparo.baque@unesum.edu.ec \\ bibi_buri@hotmail.com
}

Correspondencia: rosa.pinargote@unesum.edu.ec

I. Licenciada, Carrera de Enfermería, Facultad Ciencias de la Salud, Universidad Estatal del Sur de Manabí. Campus Los Ángeles Km.1 vía a Noboa. Jipijapa, Manabí, Ecuador.

II. Licenciada, Carrera de Enfermería, Facultad Ciencias de la Salud, Universidad Estatal del Sur de Manabí. Campus Los Ángeles Km.1 vía a Noboa. Jipijapa, Manabí, Ecuador.

III. Licenciada, Carrera de Enfermería, Facultad Ciencias de la Salud, Universidad Estatal del Sur de Manabí. Campus Los Ángeles Km.1 vía a Noboa. Jipijapa, Manabí, Ecuador.

IV. Licenciado en enfermería, Distrito 22D02 Orellana, Loreto, Salud "Parroquia García Moreno", Universidad Estatal del Sur de Manabí, Jipijapa, Manabí, Ecuador.

V. Médico Cirujano, Centro de Atención Dermatológica Integral "Cadermint", Universidad Laica Eloy Alfaro de Manabí, Manta Ecuador.

VI. Médico Cirujano, Distrito de Salud 13D09; Responsable de Promoción de la Salud e Igualdad, Universidad Laica Eloy Alfaro de Manabí, Manta Ecuador. 


\section{Resumen}

Las condiciones ambientales y la leishmaniasis ocasionan un problema de salud pública, una alteración de los factores ambientales posibilitan la transmisión del agente causal que conlleva a contraer esta enfermedad; el campo que se realizó esta investigación es en el área de la enfermería desde la perspectiva de epidemiologia mediante un estudio de tipo descriptivo no experimental y transversal, mediante análisis documental, a través de la base de datos proporcionados por el departamento de epidemiologia del Distrito de Salud $N^{\circ} 13$ D03 del Centro de Salud Jipijapa, así también los métodos de investigación como las encuestas aplicadas a los 44 pacientes que contrajeron la enfermedad. El presente estudio surge ante la presencia de casos de leishmaniasis y su relación con la alteración de factores ambientales; cuyo objetivo principal fue determinar las condiciones ambientales y leishmaniasis en el Cantón Jipijapa y según el análisis de metodología, la alteración climática, falta de conocimiento sobre leishmania, prevención de la misma y riesgos en la población de padecer esta zoonosis. Mediante los instrumentos aplicados se concluye que la mayoría de los pacientes que fueron diagnosticados positivos presenta un nivel de conocimiento bajo sobre la enfermedad y las medidas de prevención adecuadas, en el que las alteraciones ambientales tienen una gran influencia e interacción directa a la propagación de esta patología, puesto que favoreció la reproducción de vectores en especial del genero lutzomyia facilitando así la transmisión de leishmaniasis.

Palabras claves: Lutzomyia, zoonosis, enfermedades vectoriales, prevención. 


\section{Abstract}

The environmental conditions and leishmaniasis cause a public health problem, an alteration of the environmental factors allow the transmission of the causal agent that leads to contracting this disease; the field that was carried out this research is in the area of nursing from the perspective of epidemiology through a non-experimental and transversal descriptive study, through documentary analysis, through the database provided by the epidemiology department of the District of Health No. 13D03 of the Jipijapa Health Center, as well as research methods such as the surveys applied to the 44 patients who contracted the disease and interviews with health personnel. The present study arises in the presence of cases of leishmaniasis and its relationship with the alteration of environmental factors; whose main objective was to determine the environmental conditions and leishmaniasis in the Canton Jipijapa and according to the analysis of methodology, climatic alteration, lack of knowledge about leishmania, prevention of it and risks in the population of suffering from this zoonosis. Through the applied instruments it is concluded that the majority of patients who were diagnosed as positive have a low level of knowledge about the disease and adequate prevention measures, in which the environmental alterations have a great influence and direct interaction with the spread of this disease pathology, since it favored the reproduction of vectors especially of the lutzomyia genus thus facilitating the transmission of leishmaniasis.

Keywords: Lutzomyia, zoonosis, vector diseases, prevention. 


\section{Introducción.}

Según la Organización Mundial de la Salud esta patología estadísticamente se presenta con mayor prevalencia en zonas tropicales y subtropicales a nivel mundial, encontradas en países como Asia, Oriente Medio, norte y este de África, el Sur de Europa y América Central y Sudamérica. Es endémica en 88 países, 72 de los cuales están en vía de desarrollo. Se deduce que perjudica a más de 12 millones de personas en el mundo con un cálculo de 1.5 a 2 millones de casos nuevos anuales (1). Se considera una de las 9 enfermedades olvidadas, pero de gran impacto que esta aumentado debido a las condiciones ambientales generadas por el hombre, con mayor vulnerabilidad al ser humano que como consecuencia incrementa su morbi-mortalidad.

Los factores ambientales existentes ocasionan problemas de salud en nuestro diario vivir, bienestar y calidad de vida debido a los elementos químicos, sociales y psicológicos que continúan aumentando a gran nivel, debido a la profanación de contaminantes ocasionados por el hombre que en la actualidad como personas no hemos podido manejar debido al factor socioeconómico (2). La mayoría de estas determinantes ambientales son ocasionadas por el hombre puesto que no concientiza el riesgo que produce a los habitantes.

Las modificantes climáticas y socioeconómicas aumentan la fragilidad de los cambios ambientales la cual debilita el estado inmunológico de las personas puesto que propician el riesgo de contagio de patologías infecciosas y agravar el estado de la vulnerabilidad en los habitantes (3). El aparecimiento de ciertas patologías son indicadores al cambio y acrecimiento de las tasas de incidencia y enfermedades producidas por vectores, debido al cambio en los ecosistemas incitas al desplazamiento de huéspedes debido a la alteración de su morada y como consecuencia alteran nuestra calidad de vida. 
En el Ecuador la profanación que contamina nuestro entorno se produce al mal estado del transporte gubernamental y exclusivo añadiendo a esto el tráfico de vehículos que mediante los gases tóxicos generan el cambio climático y contaminación ambiental. Los vapores perjudiciales tienen un contacto directo con nuestro entorno produciendo daño en la capa de ozono a escala mundial (4). Los avances industriales en la actualidad, los medios vehiculares son utilizados a base de combustiones dañinas para nuestros ecosistemas la cual ocasiona el deterioro de salud en los habitantes ecuatorianos y también produciendo la alteración climática, generando así patologías vectoriales, cardiovasculares y metabólicas.

La leishmaniasis es una patología de origen parasitaria de gran impacto que se relaciona con el factor socioeconómico, el protozoo del genero Leishmania que ocasiona daño al ser humano, según estadísticas más de 20 especies influyen en salud, su cuadro clínico depende de la especie y el sistema inmunológico del huésped, entre las categorías de dicha enfermedad tenemos a forma visceral considerada la más grave que puede ocasionar la muerte y según la literatura se conoce como leishmaniasis visceral (LV), encontramos también aquella que afecta daño en nuestra piel conocida como leishmaniasis cutánea (LC), y por último la que produce deterioro en las mucosas llamada leishmaniasis moco cutánea (LMC) (5). De la misma manera depende de la replicación del parasito en la célula diana.

La enfermedad tiene una menor incidencia en zonas donde la temperatura es fría, en la que el ambiente controla y reduce el emporio vectorial (6), que por lo general aparece en territorios donde su clima es cálido y húmedo como la costa ecuatoriana por la biodiversidad de las regiones tropicales y montañosas. 
Las condiciones ambientales y la Leishmaniasis están directamente vinculadas y se estima estadísticamente casos reportados en la India, Nepal, Sudan y Países Bajos. En la Costa Mediterránea tiene un gran impacto debido a su aumento con relación al VIH-SIDA (7). En América está presente el tipo de Leishmaniasis visceral en Estados Unidos, Argentina y Brasil. Y como leishmaniasis cutánea ocasionando deformidades en la piel y con mayor incidencia de morbilidad está en presente en el Perú, Ecuador, Chile y Uruguay.

De acuerdo al Ministerio de Salud Pública del Ecuador (MSP) y sus estadísticas registran un acercado porcentaje al número de casos anuales con un aproximado de 1500 casos en las regiones de la Costa, Amazonia y provincias andinas. Respecto a la distribución geográfica de los vectores no se conoce con exactitud la cual indica que aumentara la incidencia de nuevos contagios en ajenas regiones del país (8). A causa de la contaminación ambiental las patologías de origen vectorial incrementaran en el futuro.

En la actualidad en la provincia de Manabí aparece con gran frecuencia, en estos últimos meses en zonas urbanas, mientras que en la antigüedad eran frecuentes en las zonas campestres o rurales debido al factor socioeconómico. Con un gran porcentaje de casos de leishmania cutánea, considerada una patología endémica, cuyo cuadro clínico principal es ulceras indoloras en la piel. La patología representa un problema de salud ya que está presente en 23 de las 24 provincias ecuatorianas según registros de la Coordinación Zonal 4 en el año 2015 se presentaron 249 casos y 235 hasta el mes de septiembre del 2016, con una mayor incidencia en la provincia de Santo Domingo de los Tsáchilas (9).

Por desconocimiento de los pacientes al inicio de la patología no acuden de forma adecuada a una consulta médica en los Centros de Salud más cercanos y como consecuencia su tratamiento es 
tardío, lo que genera complicaciones en su sintomatología, y produce un problema de gran magnitud por el presupuestó que requieren del gobierno para su oportuno cuidado y atención. En el cantón Jipijapa esta patología se ha extendido a nuestras áreas, debido a los cambios ambientales, según el Distrito de Salud 13Do3 Jipijapa - Puerto López, se habían detectado casos autóctonos de esta enfermedad en el año 2017 cuyos primeros reportes fueron registrados entre el 14 y 18 de septiembre.

La presentación de casos relacionados epidemiológicamente, es de aparición súbita y diseminación no localizada en un caso específico, la cual es un indicador de ahí transmisión activa de la enfermedad, por lo que se hacen necesario reevaluar las medidas y acciones de pesquisa de casos en la comunidad, así como las medidas de control ambiental y domiciliario.

\section{Material y metodos}

El campo que se realizó esta investigación es en el área de la enfermería desde la perspectiva epidemiológica en los pacientes con leishmaniasis, siendo un problema de salud que afecta a la población del cantón Jipijapa. Se realizó un estudio de tipo descriptivo no experimental y transversal, mediante análisis descriptivo, de frecuencia y porcentaje para las variables categóricas, se cumplió con todas las normas éticas establecidas, salvaguardando el derecho de la privacidad y consentimiento de los participantes.

Como criterio de inclusión se instauraron a los 44 pacientes con leishmaniasis, en edades comprendidas de 27 a 59 años con mayor índice, la recolección de datos se basó: en la técnica de análisis documental, a partir de la información brindada por el departamento de control Epidemiológico. También se obtuvieron datos mediante las encuestas aplicadas a los pacientes con leishmaniasis, como criterio de exclusión, pacientes que no cumplían con los requisitos para ingresar 
al programa sistema de vigilancia epidemióloga, los mismos que fueron descartados en un total de 34.

Las variables de estudio fueron las condiciones ambientales y leishmaniasis en el cantón jipijapa, con los siguientes indicadores; características ambientales, prevención, factores de riesgo, características de su diario vivir, recomendaciones de suspicacia. El universo de estudio está compuesto por los 44 pacientes que fueron diagnosticados y confirmados mediante prueba de laboratorio con leishmaniasis en el cantón Jipijapa, como la población es reducida no se aplicó formula de muestreo.

La recolección de datos se basó en encuesta, cuya función específica es dar a conocer datos relevantes para la investigación, la cual se realizó a los 44 pacientes con leishmaniasis, para obtener información verídica y confiable para el cumplimiento de esta investigación. Todos los datos fueron procesados por el programa de Excel 2010.

\section{Resultados.}

Basándose en los objetivos planteados se procedió al análisis de los resultados obtenidos de las encuentras en las que se determinó el nivel de conocimiento de las 44 personas con diagnóstico de leishmaniasis encontrándonos que el $64 \%$ de ellos desconoce acerca de la enfermedad que se relaciona al déficit de autoeducación; mientras que el 25\% de los usuarios tienen poco conocimiento de la Lutzomyia; sin embargo, existe el $11 \%$ indica conocer sobre la leishmaniasis.

Así también, se verifico si los afectados tomaron las respectivas medidas de prevención contra los vectores en donde obtuvimos que el $48 \%$ de los pacientes utilizaban los sahumerios como medio de protección, mientras que el $25 \%$ no esgrime ningún método de seguridad contra los 
vectores de la leishmania, el $18 \%$ utiliza una forma de prevención mediante los repelentes corporales, de igual manera el 9\% de la población estudiada refiere utilizar sustancias químicas a manera de insecticidas como medidas de defensa.

En cuanto a la asociación entre las condiciones ambientales y los casos de leishmaniasis se encontró que el 55\% de los pacientes realizan su jornada laboral en la zona rural, la cual fue un factor predisponente para contraer la enfermedad; mientras que el $30 \%$ de los mismos nos manifiesta que su trabajo lo realiza en la zona urbana; sin embargo, el $16 \%$ muestra que cumple jornadas laborales en las áreas urbana-marginal, lugares que tienen contacto con vegetación exuberante.

Según los determinantes ambientales que influyeron en ellos, se detalla que el 75\% viven en un ambiente cálido, a diferencia del 14\% que habita en lugares húmedos; mientras que el $11 \%$ de personas encuestadas reside en un entorno cálido y húmedo de acuerdo a las características físicas que ellos conocen.

Dentro de las mismas alteraciones del ecosistemas, la contaminación más frecuente en el habitad es la quema de basura según especifica el 52\% de los usuarios a quien se les realizó dicha encuesta; de la misma manera el 18\% nos ostenta que la profanación inadecuada de su residencia se debe a la deforestación; el 16\% exhibe que su alteración es debido a la contaminación del agua; mientras que el $14 \%$ de las personas encuestadas refiere que tiene otro tipo de contaminación cerca de su residencia.

Para identificar el factor de riesgo y las características generales de la población de estudio se da a conocer los datos e influencia de la enfermedad en los pacientes encontrando la edad como uno de los principales, donde el índice mayor que contrajo la patología fue en una escala entre 27 y 59 
años de edad correspondiente al 52\% de los habitantes a quien fue aplicado dicho cuestionario, las edades comprendidas entre 19 a 26 años indicó que el 20\% padecieron dicha enfermedad, las personas >60 años reflejó el 14\% de contagio; mientras que las períodos asociados entre 12 a 18 y de 6 a 11 años de edad representa el 7\% con igualdad de resultados; sin embargo el 0\% de los niños menores de cinco hasta cero años de edad, no manifestaron ningún caso debido al mayor cuidado por parte de sus padres.

De los 44 pacientes el $68 \%$ pertenece al género masculino los mismos que por su actividad laboral y falta de conocimiento sobre medidas de prevención contra la leishmania se encontraban en riesgo, mientras que, con un menor índice dentro de la población estudiada, el $32 \%$ corresponde al género femenino.

Otro factor predisponente a esta patología es el contacto directo con animales domésticos, el $36 \%$ nos informó que tienen una relación diaria con caninos, del mismo modo el $34 \%$ de las personas a quien se aplicó dicho instrumento de investigación nos manifestó que no tienen ningún tipo relación con mascotas, y el 25\% de los usuarios detalla que la convivencia de ellos es con felinos, mientras que el $5 \%$ de los habitantes mencionó que tiene contacto directo con las aves (loros).

\section{Discusión.}

Esta investigación se realizó en el "Centro de Salud Jipijapa” con la finalidad, de determinar el nivel de conocimiento de los pacientes sobre leishmaniasis, en la que se demostró que el mayor porcentaje de ellos no tiene conocimientos sobre la patología, la cual juega un papel predisponente en la que su forma de prevención, muchas veces no es la adecuada, según lo muestra el análisis ya realizado. 
Las acciones concretas por parte del Ministerio de Salud Pública y el área de Vigilancia Epidemiológica, brindan una atención médica gratuita, tratamiento oportuno durante los días establecidos por los protocolos y mediante el centro epidemiológico fumigación intradomiciliarios, entre las que se recomendó: utilizar ropa adecuada de colores claros y que cubran la mayor parte de su cuerpo, utilizar repelentes corporales para reducir picaduras por mosquitos y toldos para reducir el índice de leishmania y degradar la maleza 20 metros alrededor del hogar para evitar el asentamiento del vector cerca o dentro del domicilio, lo cual pueda propagar esta enfermedad a sus habitantes.

Los cambios en las condiciones ambientales, sean naturales o inducidos por el hombre, pueden aumentar o reducir la incidencia de la enfermedad. Entre los cambios ambientales que pueden modificar la incidencia de leishmaniasis se encuentran la urbanización, que es muy frecuente en países latinoamericanos como Ecuador.

La influencia de las condiciones ambientales y los casos de leishmaniasis en el Cantón Jipijapa, varían de acuerdo a su zona urbana o rural de residencia o trabajo, en los casos de leishmania positivo, la mayoría de casos se encuentran en la zona rural debido a la vegetación que existe la cual es un factor importante para que se desarrolle y proliferen los vectores.

La Bioecología del vector es semi-húmedas, que por lo general sucede después del periodo invernal, en los meses de abril y mayo que favorece el desarrollo y reservorio de la Lutzomyia, en donde lugares húmedos y con vegetación exuberante son el ambiente adecuado de estos vectores, según nos indica el estudio realizado la mayoría de los casos presentes ocurrieron en las zonas rurales, se dieron casos en la zona urbana debido a que los pacientes realizan su jornada laboral en el campo y luego se dirigían a la ciudad donde habitan. 
La emigración de las personas de las zonas rurales a la zona urbana, sea por factor trabajo. Otra razón es que, en ciertas zonas de la ciudad debido a la vegetación presente durante todo el año con humedad relativa provocado por el hombre, o personas que llevan reservorios como mascotas la cual produce el contagio.

Las situaciones donde hay mayor contagio debido a su vulnerabilidad en el cantón Jipijapa las zonas con mayor debilidad a contraer esta enfermedad son las parroquias de Sancán, Santa Rosa y Chade que son las zonas rurales más cercanas de la ciudad la cual se han importado a la zona urbana debido al factor socioeconómico. A pesar de que entre las comunas la deforestación está presente en ellas en algunas situaciones epidemiológicas, la destrucción del habitad silvestre de vegetación puede reducir la transmisión de leishmaniasis, sin embargo, en algunos casos la deforestación parece haber aumentado la infección humana y al identificar el factor de riesgo y las características generales de la población de estudio el estilo vida y el factor socioeconómico influyen en la transmisión de esta zoonosis

Las condiciones ambientales al ser modificables por la contaminación, ocasiona un desequilibrio en la cadena epidemiológica la cual produce que se ocasión una alteración en el proceso salud - enfermedad. Entre el análisis realizado nos indica que el grupo con mayor vulnerabilidad fue la etapa adulta y sus caracterices generales como: la higiene en su habitad, el uso de mascotas y la inadecuada protección en su lugar de trabajo se pudo evidenciar que existen falencias en la población estudiada al momento de prevenir esta enfermedad de origen emergente.

\section{Bibliografia.}

1. Sanchez, Leonardo; Saenz, Elena; Mendoza, Julia. In Educacion Medica Continua; 2014; Madrid. p. 2. 
2. Almedia ZP. Analisis comporativo del nivel de conocimiento sobre leishmaniasis cuanea entre el personal medico de zonas medicas del primer nivel de salud. tesis. Santo Domingo: Pontifica Universidad Catolica del Ecuador, Ciencias; 2016.

3. Sandry K. Ssaud delas Americas. [Online].; 2017 [cited 2017 febrero 9. Available from: http://salud.america.com.

4. Chiquinga F. Determinantes de Vectores de Lishmaniasis. Tesis. Guaranda - Ecuador: Universidad de Bolivar, Ciencias; 2013.

5. Lopez, Cespedes, Angeles. Diagnostico y seroprevalencia de Lishmaniasis. Tesis Doctorial. Mexico: Universidad de Grranada, Parasitologia; 2013.

6. Andrade M. Enfermedades tropicales en el Ecuador. Tesis. Cuenca: Universidad Catolica de Cuenca, Ambiente; 2014.

7. Tene E. Prevalencia de leishmaniasis en el area II de pastaza, asociadas a Factores de Riesgo. Tesis. Ambato: Universidad Tecnica de Ambato, ciencias; 2015.

8. Correa M. Centralizacion y diagnostico de laboratorio de leishmaniasis cutanea. Tesis. Babahoyo: Universidad Tecnica de Babahoyo, ciencias; 2014.

9. Cruzete M, Andes. Costo beneficio de atencion de leishmaniasis. Tesis. Portoviejo: Universidad Tecnica de Manabi, Ciencias; 2016. 